Volume 117, Number 1

tribution in the methods section. We acknowledge that the inference that "a DHCA + RCP period of up to 80 minutes under a nasopharyngeal temperature of $18^{\circ} \mathrm{C}$ is safe" in our experience is rather anecdotal and cannot be totally substantiated. However, if we divide the patients into 2 groups - those who had DHCA + RCP for less than 60 minutes and those in whom this period lasted 60 minutes or more-we cannot find any difference in the incidence of mortality, stroke, and transient neurologic dysfunction. However, it is obvious that we should reduce the period of DHCA as much as possible, even in patients who require a complex repair of the aortic arch.

Yutaka Okita, $M D$

Shinichi Takamoto, $M D$

Motomi Ando, $M D$

Yasunaru Kawashima, MD

Department of Cardiovascular Surgery National Cardiovascular Center

Osaka, Japan

12/8/93654

\section{Reconstruction of the aortic valve with autologous pericardium: An experimental study}

To the Editor:

Valve repair has several advantages over prosthetic valve replacement, including low morbidity and mortality and lower risk of thromboembolism, hemorrhage, and septic endocarditis. Different techniques of mitral valve repair have been described, with good results.' Unfortunately, the results of aortic valve repair are not as favorable. In this report I describe a new technique of aortic valve reconstruction with an autologous pericardial patch, which my colleagues and I have used.

In 5 cadaver hearts, autologous pericardium was fixed in $0.6 \%$ glutaraldehyde solution for 10 minutes. $^{2}$ An aortotomy was used. Pericardium was cut to simulate the dimensions of the valve to be repaired on the basis of the concept of the aortic root geometry as a truncated cone. ${ }^{3}$ The diameter at the highest point of attachment of the leaflets (sinotubular diameter) is about $20 \%$ less than the diameter at the inlet (surgical anulus diameter). During systole the sinotubular diameter increases while the inlet diameter decreases, changing the root geometry from conical to cylindrical. The reverse occurs in diastole, at which time the leaflets tilt toward the ventricle. ${ }^{4}$

The noncoronary cusp is fashioned first. It is measured at its attachment line and height with a soft wire (Fig $1, A)$ and the measurements are marked on the pericardium (Fig $1, B$ ). The resulting " $a-b$ " is the length at the sinotubular circumference but not the true length of the cusp. Therefore, we lengthened it to the size of its projection into the surgical (inlet) circumfer-

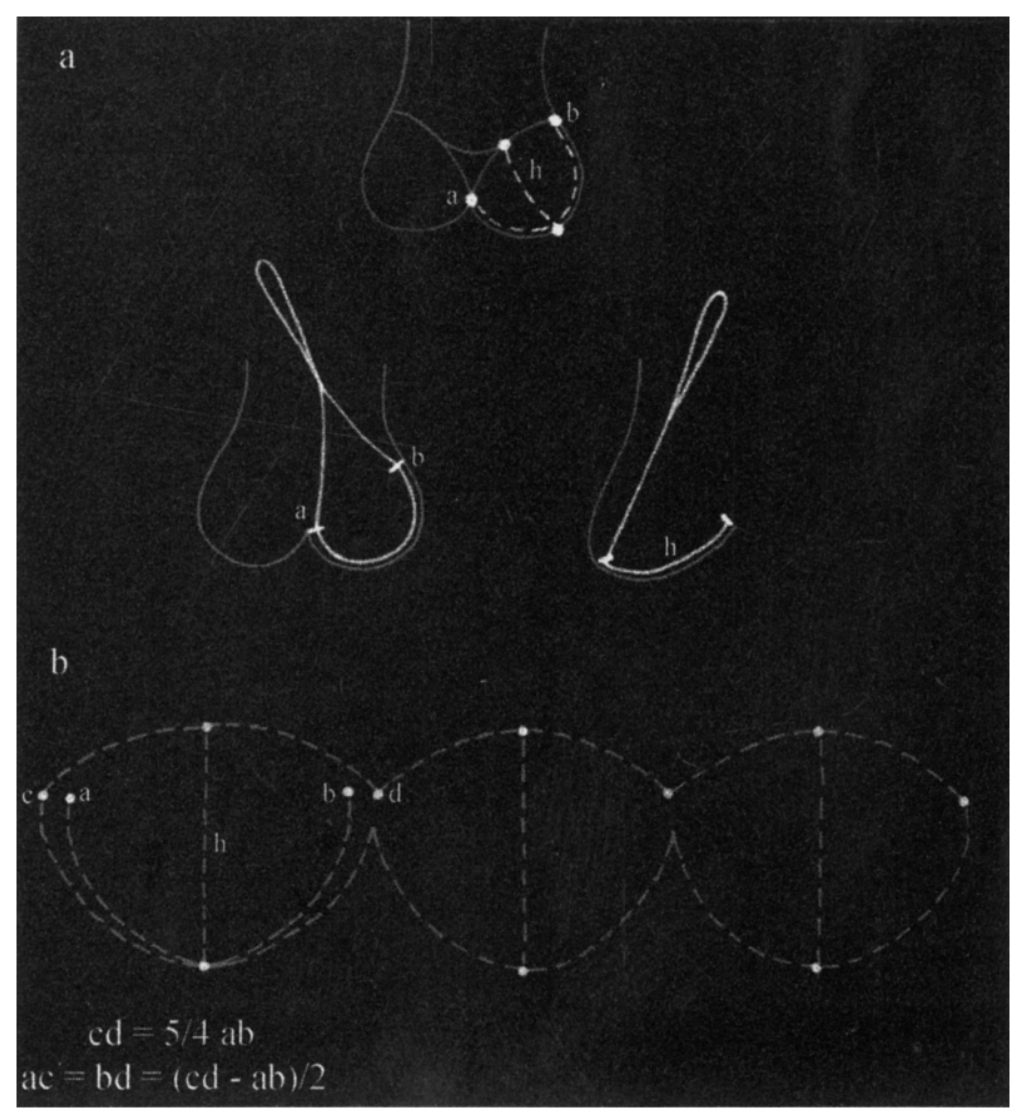

Fig 1. Cusp size measurements and fashioning of the pericardial patch. A, Taking of the noncoronary cusp attachment line and height measurements with the soft wire. B, Marking of the sizes on the pericardium and fashioning of the pericardial patch. 


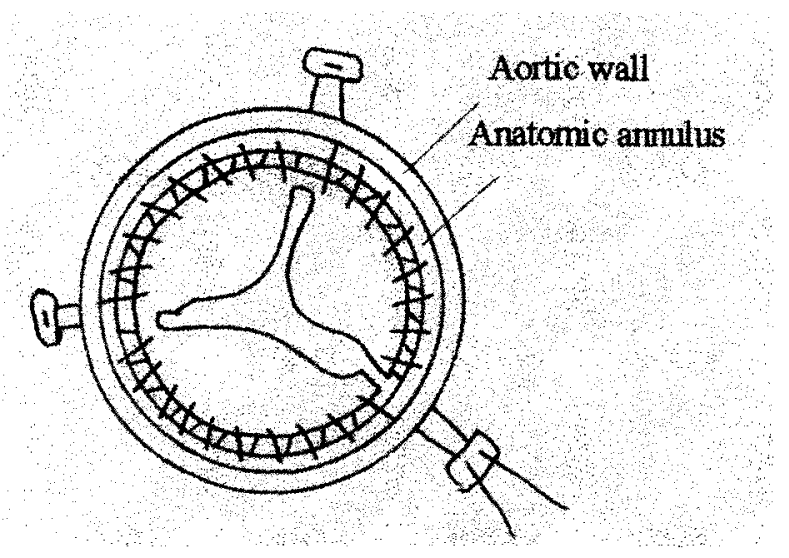

Fig 2. Suturing of the pericardial patch to the semilunar lines of the leaflet attachments with continuous 4-0 Prolene suture and reinforcing of the commissural zones with Dacron pledgets from outside.

ence, which is $20 \%$ more, as mentioned earlier (Fig 1, B). Indeed, the length of each cusp has to be equal to its projection into the inlet (annular) circumference to be able to straighten during systole when the change from the conical form to the cylindrical one occurs. The tilting of the cusps toward the aortic wall with more complete opening of the valve becomes possible because the inlet diameter at the anulus decreases. Changing the cylindrical form of the root to a conical one in diastole will make coaptation of the cusps possible.

The coronary cusps are fashioned similarly and marked on pericardium. The pericardial patch is tailored along the marked line, leaving 2 to $3 \mathrm{~mm}$ of the tissue for the sutures (Fig 1, B).

The next step is removing the native valve and suturing the pericardial patch to the semilunar lines of the leaflet attachments (anatomic anulus) with continuous 4-0 polypropylene suture (Prolene; Ethicon, Inc, Somerville, NJ), which is passed out through the aortic wall in the commissural zones and reinforced with Dacron pledgets from the outside (Fig 2). Then we place additional mattress sutures in the commissural zones, passing them out through the aortic wall and the same pledgets.

To check valve competence after reconstruction in the cadaver heart, we put a purse-string suture through the aortic wall along its circumference and subject this to hydrostatic presssure. After incising the left ventricular apex and opening the ventricle, we could observe the newly created aortic valve from below. Complete coaptation of the leaflets with no regurgitant jet and competent suture lines along the leaflets attachment were observed in all 5 cases. We also could observe good filling of all coronary arteries.

We concluded that our technique of aortic valve reconstruction with an autologous pericardial patch should be tested in animal experiments and then possibly be tried in a clinical application in the future as an alternative to mechanical or prosthetic biologic valve implantation and/or as an intermediary procedure in young patients, postponing prosthetic valve replacement.
The prerequisite to our method is the absence of significant distortion of aortic root geometry in hearts with severely diseased valves.

We wish to pursue this study further and look forward to collaborating with others in this endeavor.

Vahe C. Gasparyan, $M D$

Department of Surgery

Garrison Hospital of Yerevan

26 Papazaian st 24, 375012

Yerevan, Republic of Armenia

\section{REFERENCES}

1. Carpentier A. Cardiac valve surgery-the "French" correction." J Thorac Cardiovasc Surg 1983;86:323-7.

2. Chachques JC, Vasseur B, Perier P, Balansa J, Chauvand S, Carpentier A. A rapid method to stabilize biological material for cardiovascular surgery. Ann N Y Acad Sci 1998;529:184-6.

3. Sarsam MAI, Yacoub M. Remodeling of the aortic valve anulus. J Thorac Cardiovasc Surg 1993;105:435-8.

4. Thubrikar M, Pieperass WC, Shaner TW, Nolan SP. The design of the normal aortic valve. Am J Physiol 1981;241:H795-801.

$12 / 8 / 94054$

A stable model of chronic bilateral ventricular insufficiency (dilated cardiomyopathy) induced by arteriovenous anastomosis and doxorubicin administration in sheep

To the Editor

Congratulations to Toyoda, Okada, and Kashem' on their recent article, "A Canine Model of Dilated Cardiomyopathy Induced by Repetitive Intracoronary Doxorubicin Administration." We need such a simple and reproducible animal model of stable, nonreversible chronic heart failure if we are to evaluate new surgical treatments for pre-end-stage congestive heart failure. The authors described cannulation of the coronary artery via the femoral artery and transcatheter infusion of doxorubicin, which resulted in visible signs of left ventricular insufficiency after 5 weekly doses of intracoronary doxorubicin; however, they reported that "the right ventricle seemed to be affected only slightly by doxorubicin." Especially for cardiomyopathy research, one needs to create a model of bilateral heart failure.

In a recently completed (unpublished) investigation involving adult sheep, we created a bilateral model through first inducing right ventricular insufficiency by a surgical anastomosis between the right jugular vein and right external cardiac artery. After 1 month, we observed initial signs of insufficiency in the right ventricle (Table I) but not in the left. At this point, we began daily administration of (intravenous, not intracoronary) doxorubicin, which continued for the next 4 weeks and resulted in left ventricular insufficiency (Table II).

Please note that we made no attempt to determine whether the anastomosis or doxorubicin had the greater effect in inducing right ventricular insufficiency, although Toyoda, Okada, and Kashem observed that 5 weekly doses of the drug were insufficient for the purpose. On the other hand, Smink and colleagues ${ }^{2}$ induced quite stable bilateral ventricular insufficiency after an anastomosis was in place for only 3 months. 